\title{
Discussion on the Cultural Implication and Social Humanity Function of Medical Terms in Doctor- Patient Communication
}

\author{
Wei Liu ${ }^{1}$ Hongping Cheng ${ }^{1}$ Wenjuan Lin ${ }^{1, *}$
}

${ }^{1}$ Center of Health Administration and Development Studies, Hubei University of Medicine, Shiyan, Hubei 442000, China

*Corresponding author. Email: autumnwhisper@163.com

\begin{abstract}
Based on the discussion of the pragmatic functions of medical terms, this paper examines the social humanity functions of medical terms in doctor-patient communication, explores the humanity meaning of medical terms themselves, gives full play to the due humanity value of medical terms, and promotes active and effective doctor-patient communication. In doctor-patient communication, medical terms can be viewed as the marker of social identity for doctors, which function as the education and popularization of medical knowledge. Medical terms are also regarded as the marker of the transformation of medical socialization, coupled with politeness and tenderness in doctor-patient communication. With proper and effective use of medical terms in doctor-patient communication, the positive social humanity functions mentioned above can be exploited at their best, as a result of which gets access to medical humanism and promotion of the relationship between doctors and patients.
\end{abstract}

Keywords: medical terms, doctor-patient communication, pragmatical functions, humanity

\section{INTRODUCTION}

The medical term is a language that accurately describes the human body and its related parts, conditions, processes and procedures in a sciencebased way, and is used in the fields of medicine and nursing. Chinese and foreign scholars' research on medical terms mainly focuses on the following two aspects: one is pragmatics research. That is, through systematic sorting and classification, medical terms are integrated into a vocabulary system that is highly academic and professional, rigorous in expression, and scientific and accurate for medical workers and medical students to learn, reference, standardize and use. The second is etymology research. These studies mainly explore its own word formation characteristics and expression methods from the perspective of etymology, and on this basis, to explore its cultural origins and language thinking characteristics. As for the use of medical terms in doctor-patient communication, the

*Funds: This article is the 2017 Hubei Medical College Health Management and Health Development Research Center Development Fund Project (No. 2017ZD004) and 2017 Hubei Provincial Department of Education Humanities and Social Sciences Research Project "Comparative Study of Chinese and Western Parataxis and Hypotaxis Medical Terms" Research Results (No. 17G150). consensus among scholars is: it's necessary to try to avoid or reduce the use, and replace it with plain language. Therefore, in the research field of doctorpatient communication, medical term is either disregarded as a type of medical language that has been concluded, or occasionally mentioned as a negative factor of doctor-patient communication barriers and communication skills. In short, in the research of doctor-patient communication, medical terms have been "kept away" because of its scientific, accurate, standardized, and rigorous characteristics. And its due humanity significance, function and value in the communication between doctors and patients have also been seriously ignored.

\section{THE SOCIAL HUMANITY FUNCTIONS OF MEDICAL TERMS IN THE DOCTOR- PATIENT COMMUNICATION}

The medical term is a linguistic variant of conversational language at the vocabulary level in daily expressions, a language symbol of medical concepts, and a carrier of medicine and doctorpatient communication. The medical term has its specific pragmatic function. On the basis of reflecting scientificity and rigor, it also carries a 
certain humanity meaning. In doctor-patient communication, the pragmatic function of medical terms is not just a scientific, objective, and accurate expression, and some communication obstacles or failures caused by this can't deny the humanity meaning and other positive pragmatic functions carried by it. In doctor-patient communication, this is a cultural indifference and prejudice to medical terms.

\section{A. Educational popularization function of medical terms}

Medical terms are based on medical scientific concepts, accurately and strictly reflecting the concept and the characteristics of the disease and symptoms of the disease, and the concept expressed is specific, it is an entity with a clear semantic scope, and it is a kind of specific and precise expression, a specialized vocabulary to express the scientific connotation and essential attributes of a certain concept, and an embodiment of the rigorous medical research. Doctor-patient dialogue first needs language as a communication tool to achieve the purpose of inquiries. The primary purpose of doctor-patient communication is to obtain information about the diagnosis of diseases, to describe and record the routine and special life states of patients through the use of professional, scientific and accurate terms, to understand the laws of life, to grasp the nature of diseases, and to form scientific and rigorous diagnosis results. When transmitting disease information, the use of professional medical terms reflects the scientific and professional nature of medical events. For example, "clinical death" and "biological death" are concepts with different meanings. The different diagnoses of "disintegration of bones" and "fracture" have the effect of education and popularization of medical knowledge for patients to understand the state of an illness. "Cervical columnar epithelial ectopia" describes the normal physiological phenomenon of the cervix more accurately than the use of "cervix erosion" and eliminates the misunderstanding of the normal performance of the cervix. There are also some impediments to the use of non-rigorous terms that have caused public misunderstanding of medical knowledge. For example, "hemorrhoids" are not sores, but a varicose vein lump; the "lymph cancer" should be "lymphoma", and a malignant lymphoma is cancer. "Decayed teeth" are saprodontia caused by dental plaque, not caused by being damaged by worms. Although the descriptions of "being flustered" and "feeling suffocated" are vivid, they are not as accurate as the expressions of "palpitations" and "dyspnea". Standard medical terms help people correctly understand medical knowledge, increase medical knowledge, and reduce people's misunderstandings about doctors' diagnosis and treatment. Therefore, the use of medical terms should have the educational popularization function of medical culture.

\section{B. Humanity communication functions of medical terms}

The use of medical terms in doctor-patient communication can reflect professional respect for patients, objectively convey information in communication, and psychologically eliminate the patient's known disease burden, embodying the humanistic feelings of "the benevolence of doctors" and having the function of politeness and warmth. The use of medical terms in doctor-patient communication also has the function of euphemism and harmony. While easy-to-understand medical expression can eliminate information barriers in doctor-patient communication, the psychological burden and direct harm caused to patients are also obvious. A situation in which words fail to convey meaning can even aggravate the sense of fear or discrimination in patients, and it is not conducive to effective communication between doctors and patients. Relatively speaking, the use of medical terms has a positive euphemism function, which will make the doctor-patient atmosphere more harmonious. Medical staff must not only heal the physical pain of the patient, but also heal the psychological scar, so as to be in line with the treatment of diseases by modern medicine (Wang Jinfan, 2007). To treat the body requires medical technology, to heal the mind requires communication, and to communicate requires language. Choosing appropriate medical terms in a specific context and using them reasonably is not only a kind of respect for the profession, but also for the patients. Popularized medical terms are very beneficial to doctors, patients, and society. In the doctor-patient communication, doctors must first integrate medical concepts in professional terms, then use reasonable metaphors to visualize abstract medical examinations or medical methods, and deconstruct rigid academic terms with easy-tounderstand objects or phenomena. This not only shortens the distance between the doctor and the patient, but also increases the talkable nature of the dialogue between each other, and doesn't cause ambiguity or psychological pressure to the patient, thereby promoting the communication between the doctor and the patient (Wu Yunqian, Zhou Hongguang, 2018).

The charm of language requires doctors to grasp it in order to play its due role. A doctor's ability to express himself is as important or more important than his own medical level (Zhu Jinfu \& Li Gongying, 2016). Popular medical terms in doctorpatient communication are easier for patients to understand. It is like the FIGO staging of cervical cancer. Many non-medical patients can't understand the difference between 1234 stages. The doctor can 
explain the professional terminology to the patient first, and then explain it in plain language, which not only reflects the authority of the doctor, but also clearly explains the condition to the patient, so that the patient will not misunderstand. For another example, "convulsions" and "strokes" are easy to understand for family members. "Eclampsia" and "cerebral infarction" maybe beyond understanding of the family members.

\section{Social marker functions of medical terms}

The doctor-patient relationship is the most basic and active factor in medical behavior. This relationship is generated at a specific time and in a specific place, and is established in the dialogue between doctors and patients (Guo Jia, 2013). Therefore, doctor-patient dialogue and communication are a direct manifestation of the doctor-patient relationship, and can reflect the obvious social marks of both parties in the conversation. In doctor-patient communication, doctors first use language to achieve the purpose of communication and inquiries, and they use terms for the patient's diseases and symptoms to confirm the social group and social identity to which they belong. Therefore, the medical terms used by doctors can be used to confirm their social identity, and furthermore, they can determine the identity attributes of the subdivision groups of the social group they belong to, forming a professional variant of medical language, such as Pneumology Department, Department of Cardiology, Gastroenterology Department, Orthopedics Department and other subdivision groups. Doctors have a wealth of professional terminology. In the doctor-patient conversation, the doctor puts himself in a different language position from the patient through the use of terms, which has a higher register and highlights the social role of the doctor. Compared with the use of popular language, professional terminology is an accurate description of medical events and disease diagnosis and treatment, which can reflect the doctor's identity and theoretical knowledge system, such as upset stomach-abdominal pain; great aunt-menstruation; nervous and startled-anxiety disorder; sore throatthroat pain; the lumbar vertebra can't be straightened-protrusion of intervertebral disc; tuberculosis-phthisis; malnutritional stagnationindigestion; convulsions-eclampsia; sheep madness-epilepsy and so on; the former is an easyto-understand term that can be more easily understood by the public and serves as a way to popularize knowledge. It is a means for doctors to adapt to patient understanding and communication on the basis of grasping the concept of terminology in doctor-patient communication. The latter is a rigorous positioning and description of the nidus, and a symbol of professional status, rather than popular expressions such as "quack doctors" and "witch doctors and warlocks".

\section{Socio-pragmatic functions of medical terms}

Medical terms should not only have the characteristics of their scientific culture, that is, medical orientation, but also have the pragmatic function of its humanistic cultural orientation. The development of modern medicine and the transformation of the model, from the biomedical model to the bio-psycho-social medicine model, are accelerating the integration of humanity factors. This transformation has gradually broken out of the purely physiological and pathological thinking patterns, and it has become more and more inevitably intertwined with psychological, social and pragmatic factors (Zeng Kongsheng, 2004). Medical understanding of health and disease has been gradually established on the basis of understanding of human spiritual activities and the understanding of the relationship between humans and society. It plays a fundamental role in understanding and explaining physiology, grasping the essence of disease, accumulating and inheriting diagnosis and treatment experience, and forming and prospering medical culture (Wang Jingyan, 2009). The accurate and appropriate use of medical terms in doctor-patient communication is a shift in the socialization of medical education. The nature of medicine is humanism, serving people, and the use of medical terms to spread and popularize medical knowledge. Explaining professional medical terms in a popular way makes it easier for ordinary people to understand, which not only achieves the purpose of popularizing knowledge, promotes the social extension of the humanistic nature of medical terms, but also a measure for medical workers to protect themselves. The development and popularization of medical terms has also prompted medical language researchers and medical workers to further examine their pragmatic function and positive role in doctorpatient communication. Medical term itself should also receive sufficient humanistic care and attention. Professional terminology is a manifestation of the rigorous medical science, a very precise description of the diagnosis and treatment of diseases in medical documents, and a miniature of the development and progress of medical technology over the years. The popularization of the spectrum of medical terms is conducive to improving the medical quality of the public, is conducive to the advancement of medical knowledge to the general public, and promotes the cognition, prevention and care of diseases, which is the progress of medical socialization. 


\section{CONCLUSION}

Medicine has a dual nature. It is both a science and a human science, and it needs the nourishment of the humanistic spirit. Medical terms are the most direct reflection of these two attributes. Therefore, medical terms should not be regarded as a kind of "cold" scientific expression in doctor-patient communication, but as a cultural bridge connecting doctors and patients with positive cultural significance. For a long time, medical terms have been positioned as a scientific, accurate and standardized professional expression in doctorpatient communication, and have no communication power; they are also unconvincing because of their profoundness and difficulty in understanding. The negative influence of medical terms in the communication between doctors and patients prompts people to turn to other positive narrative rules and methods, and strive to conduct effective communication and avoid conflicts and disputes. Using as few or no medical terms as possible in doctor-patient communication doesn't completely deny their humanistic meaning and application value. The key is to analyze it dialectically and use it reasonably depending on the specific context. Medical staff and patients are each other's "aesthetic subject and object" in the communication between doctors and patients. This kind of aesthetics has a deep care for human nature, is health-centered, and is highly utilitarian. Since medical terms are the most direct way of conceptual communication in doctor-patient communication, the rational and effective use of medical terms for doctor-patient communication can balance the utilitarian mentality of both parties, naturally integrate science and emotion into medical behavior and doctor-patient relationship, and fully demonstrate the humanistic attributes and feelings of medicine. The internationalization and socialization of modern medicine continue to accelerate the integration of humanity factors and medical science. The development and popularization of medical terms should also prompt medical language researchers and medical workers to re-examine its social and humanistic functions and positive effects in doctor-patient communication. Medical terms themselves should also receive sufficient humanistic care and attention, so as to effectively improve and balance the doctor-patient relationship, and improve the efficiency and effectiveness of doctor-patient communication.

\section{References}

[1] Zeng Kongsheng. Two definitions of medical linguistics [J]. Medicine and Philosophy, 2004, 25(7): 47-48. (in Chinese)
[2] Wang Jingyan. Interpretation of medical language from the cultural perspective [J]. Chinese Medical Ethics, 2009, 22(4): 87-88. (in Chinese)

[3] Wang Jinfan. Thinking about the connotation and purpose of Chinese doctor-patient communication [J]. Chinese Hospital Management, 2007, 27(3): 28-29. (in Chinese)

[4] Zhou Xiangtao. Analysis on the significance of strengthening doctor-patient communication [J]. Journal of Jinzhou Medical College: Social Science Edition, 2006, 4(2); 14-16. (in Chinese)

[5] Luo Huiyu. Talking about the purpose and significance of doctor-patient communication $[\mathrm{J}]$. Health Vocational Education, 2011, 29 (22):154. (in Chinese)

[6] Wu Qizhen. Methods and skills of doctor-patient communication [J]. Medicine and Society, 2008, 21(2): 4849. (in Chinese)

[7] Xu Jianfeng, Wang Wenxi, Sun Zhijian. Psychological skills of doctor-patient communication [J]. Journal of Bethune Military Medical College, 2006, 4 (2): 119-120. (in Chinese)

[8] Cui Jun, Yu Hong. Talking about doctor-patient communication skills from the perspective of psychology [J]. Journal of Baotou Medicine, 2007, 31(1): 20-22. (in Chinese)

[9] Guo Jia. Research on Doctor-patient dialogue from the perspective of sociolinguistics $[\mathrm{J}]$. Journal of North University of China (Social Science Edition), 2013, 29(6): 87-91. (in Chinese)

[10] Wu Mengchao. Clinical medicine and humanistic quality [J]. Medicine and Philosophy, 2002, 23 (9): 1-4. (in Chinese)

[11] Wangzhu Jinfu, Li Gongying. Doctor-patient communication $[\mathrm{M}]$. Beijing: Higher Education Press, 2016: 58-96. (in Chinese)

[12] Wu Yunqian, Zhou Hongguang. Talking about the necessity of popularizing medical terms in doctor-patient communication $[\mathrm{J}]$. Chinese Medical Humanities, 2018,4(12):11-13. (in Chinese) 